\title{
New Trends in the Hydrodynamics of High-Speed Overwater Systems
}

\author{
V.A. Eroshin, A.V. Boyko \\ Institute of Mechanics, Lomonosov Moscow State University, \\ Moscow, 119192, Russia \\ mailband@mail.ru
}

\begin{abstract}
For a long time, the debate about the advisability of using rolling bodies on the surface of the water for transport purposes remained purely rhetorical, although the first patent was issued to D. Vanderburg in 1888 [1-4]. However, in recent years, numerous cases of water skiing on buggies, snowmobiles and motorcycles $[5,6]$ make us think about the practical use of this idea. The article describes the conditions of water rolling of wheel systems with lugs, the modeling of this phenomenon is carried out, the dependences of the draft and bearing capacity of the wheels on the Froude number are obtained for a number of values of the dimensionless mass of the system, and the position of the boundary of the stable rolling region is estimated.
\end{abstract}

Keywords: rolling on water on wheels, physical modeling, hydrodynamic forces, rolling conditions

As a result of the studies, a technique was developed to simulate the phenomenon of rolling a wheeled vehicle on a free surface of water, schemes for measuring the draft of a cylinder when towing in a hydrochannel and when rolling on a self-propelled model were proposed, and the dependences of dimensionless draft on the Froude number for various values of dimensionless mass were obtained.

The experiments showed that the bearing capacity of the wheel system at a high rolling speed does not depend on the Froude number. The approximate position of the boundary of the rolling region is found, and the conditions are established under which the vehicle can stably roll along the unperturbed free surface of the water. 
УДК 514.86

\title{
Новые тенденции в гидродинамике скоростных надводных систем
}

\author{
В.А. Ерошин, А.В. Бойко \\ НИИ механики, Московский Государственный Университет им. М.В. Ломоносова, \\ Россия, Москва, 119192, Мичуринский проспект д.1 \\ mailband@mail.ru
}

\begin{abstract}
Аннотация
Долгое время споры о целесообразности использования качения тел по поверхности воды в транспортных целях оставались чисто риторическими, хотя первый патент был выдан Д. Вандербургу еще в 1888 году [1-4]. Однако, в последние годы многочисленные случаи катания по воде на багги, снегоходах и мотоциклах $[5,6]$ заставляют нас подумать о практическом использовании этой идеи. В статье описаны условия качения по воде колесных систем с грунтозацепами, проведено моделирование этого явления, получены зависимости осадки и несущей способности колес от числа Фруда для ряда значений безразмерной массы системы, а также дана оценка положения границы области устойчивого качения.
\end{abstract}

Ключевые слова: качение по воде на колесах, физическое моделирование, гидродинамические силы, условия качения.

\section{1. Введение}

Идея качения по воде на колесах вот уже почти полторы сотни лет бродит в умах изобретателей, мечтателей и любителей острых ощущений (рис. 1). Многочисленные случаи качения по воде на багги, снегоходах и мотоциклах заставляют нас вернуться к изучению этого вопроса, а возможно, и подумать о его практическом использовании.

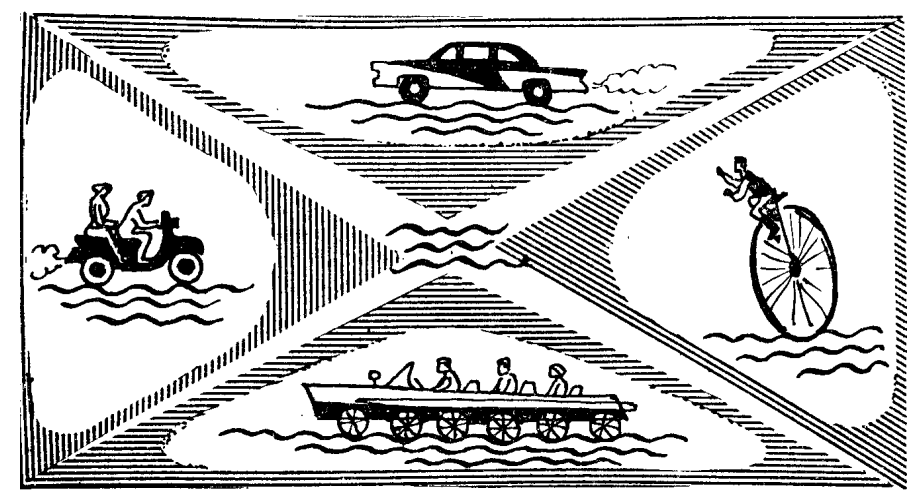

Рис. 1

Суда на колесах обладают рядом преимуществ. Прежде всего это высокая скорость качения по воде. Скорость глиссеров и судов на подводных крыльях составляет примерно $60 \div 70$ км/час, судов на воздушной подушке $-100 \div 110$ км/ч. А на колесах по спокойной воде можно катиться со скоростью $100 \div 200$ км/час и, возможно, быстрее (правда при малых скоростях они будут двигаться как водоизмещающие суда, но это отдельный вопрос выхода на «скоростной режим»). Такие суда будут востребованы при чрезвычайных ситуациях (например, наводнениях), при проведении розыскных и спасательных работ, оказании ско- 
рой и неотложной медицинской помощи в отдаленных районах страны, в том числе в условиях Арктической зоны. Таким образом, имеются многочисленные случаи качения по воде на колесах, а общей теории этого вопроса пока нет, хотя первые шаги были сделаны еще в 1965 г. [7]. В статье рассматривается качение тел по спокойной поверхности воды. Описаны условия качения колесных систем с грунтозацепами, проведено моделирование этого явления, получены зависимости осадки и несущей способности колес от числа Фруда для ряда значений безразмерной массы системы, а также дана оценка положения границы области устойчивого качения[8].

\section{2. Моделирование явления качения}

Для выяснения условий, при которых колесные транспортные средства могут устойчиво катиться по свободной поверхности воды, необходимо выбрать систему параметров, определяющих совокупность динамически подобных движений, и выделить их возможные безразмерные комбинации.

Рассмотрим качение по воде колеса с грунтозацепами (как у багги). Особенностью явления качения по поверхности воды такого колеса является то, что поддерживающая сила обусловлена динамической реакцией воды, как и при глиссировании, причем величина вертикальной составляющей гидродинамической силы практически такая же, как и при качении по воде гладкого цилиндра $[7,8]$. Однако существенным отличием таких колес является то, что они одновременно выполняют функции движителей. Транспортные средства подобного типа могут не только катиться по воде с большой скоростью, но и ходить по мелководью, а при движении на берегу не уступают внедорожникам.

Установившееся качение колесного транспортного средства по поверхности воды с большой скоростью определяется параметрами $[8,9]$

$$
V, m, D, \rho, g, B \text { и } h,
$$

где $V$ - скорость; $\rho$ - плотность воды; $g$ - ускорение свободного падения; $m$ - масса транспортного средства; $D$ и $B$ - диаметр и ширина протекторов колес; $h$ - высота грунтозацепов. При скорости движения порядка 100 км/час осадка мала по сравнению с диаметром колес $(\Delta \ll D)$, а отрыв жидкости происходит в нижней точке колес. В этом случае основными безразмерными параметрами, характеризующими качение (кроме геометрических), являются число Фруда $V / \sqrt{g D}$ и безразмерная масса $m / M$.

В плоском случае выражения для безразмерной осадки $\Delta / D$ и безразмерной несущей способности цилиндра $Y / m g$ имеют вид [8]

$$
\frac{\Delta}{D}=C \frac{(m / M)}{F r^{2}}, \frac{Y}{M g}=\frac{m}{M},
$$

где $M=(\pi n / 4) \rho D^{2} B$ - характерная масса колесной системы; $n$ - число колес; $C$ - коэффициент подобия, зависящий от формы боковой поверхности цилиндра (числа и размеров плиц, рисунка протекторов колес и т.п.).

Для гладкого цилиндра $C=1$ [7].

\section{3. Зависимость средней осадки колес от числа Фруда и массы системы}

На рис. 2 приведены зависимости безразмерной осадки $\Delta / D$ от числа Фруда для ряда значений безразмерной массы. Кружочками (кривая 1) изображены опытные данные, полученные при буксировке цилиндров с плицами в гидроканале (плоский случай, $m / M=0.12$ ). Штриховая линия - решение И.Т. Егорова [7]. Квадратики и треугольники (кривые 2,3) соответствуют опытным данным, полученным при качении тяжелой самоходной модели $\left(m_{1}=3.0\right.$ кг, $m_{1} / M=2.05$ и $m_{2}=3.9$ кг, $m_{2} / M=2.69$ соответственно). 


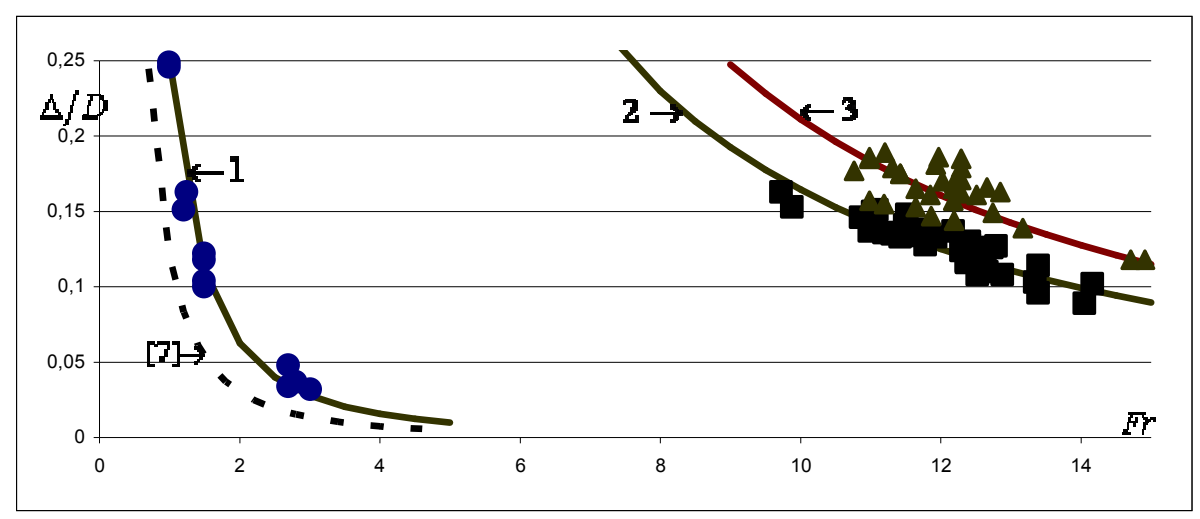

Рис. 2. Зависимость безразмерной осадки от числа Фруда

При качении самоходной модели течение не является плоским. В этом случае зависимость безразмерной осадки от числа Фруда лучше описывается формулой

$$
\frac{\Delta}{D}=C \frac{(m / M)}{F r^{3 / 2}}
$$

Как будет показано ниже, величина коэффициента подобия $C$ в формуле (2) приблизительно равна $C=2.57$.

\section{4. Оценка несущей способности колесной системы и коэффициент подобия}

Оценим величину несущей способности $Y$ 4-х колесной полноприводной самоходной модели. При установившемся движении подъемная сила, действующая на модель, равна $Y=m g(m-$ масса модели) или в безразмерном виде $Y / M g=(m / M)$, т.е. из формулы (2) получаем

$$
\frac{Y}{M g}=\frac{1}{C}\left(\frac{\Delta}{D}\right) F r^{3 / 2}
$$

На рис. 3 приведена зависимость коэффициента подобия $C$ от числа Фруда. При $0<F r<3$ опытные данные изображены кружочками $(m / M \approx 0.12)$, при $9<F r<15$ - квадратиками $(m / M \approx 2.05)$ и треугольниками $(m / M \approx 2.69)$; штриховой линией изображено среднее арифметическое значение коэффициента подобия $C=2.57$.

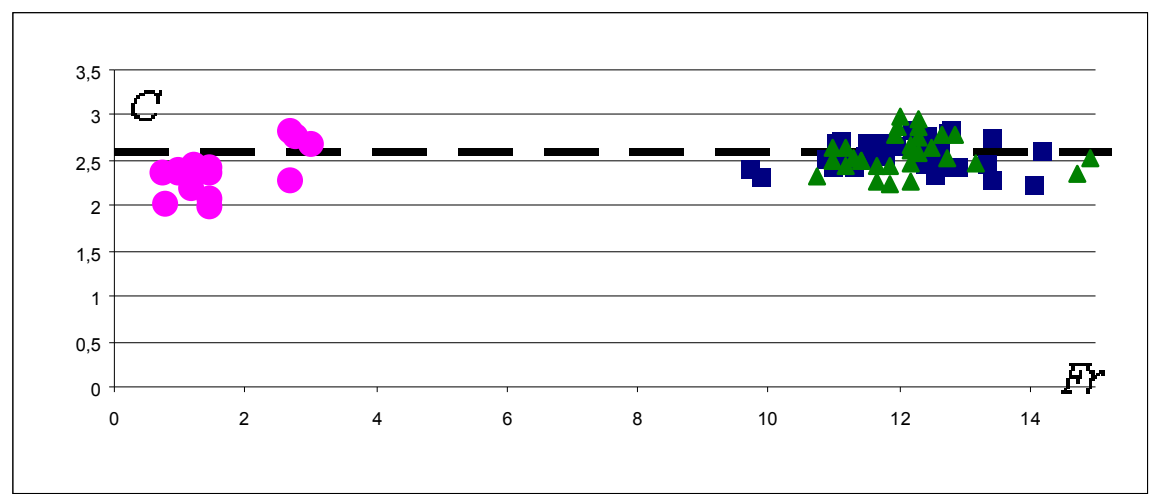

Рис. 3. Зависимость коэффициента подобия $C$ от числа Фруда 
При числах Фруда $3<F r<9$ опытные данные отсутствуют. В этой области необходимо использовать более легкие самоходные модели, которых в настоящее время нет. Имеющаяся модель в этой области не может катиться по воде (утонет).

Несмотря на большой разброс опытных данных эти эксперименты показывают, что безразмерный коэффициент $C=(\Delta / D) F r^{3 / 2} /(m / M)$, по-видимому, является универсальной постоянной при качении по воде колес данной формы с большой скоростью.

В этом случае можно найти зависимость несущей способности колес от числа Фруда. Эксперименты показывают, что рабочая осадка колес составляет примерно $0.1<\Delta / D<0.15$. При большей осадке качение становится неустойчивым и движение может перейти в режим плавания (как водоизмещающее судно). При меньшей осадке движения возможно, но скорость (число Фруда) должна быть больше.

На рис. 4 приведены зависимости безразмерной подъемной силы $Y / M g$ от числа Фруда, полученные по формуле (3) с использованием всех имеющихся опытных данных. Величина параметра $C$ при расчетах принималась равной $C=2.57$. Сплошными линиями изображены точные значения подъемной силы, которые в данном случае известны: $Y_{1} / M g \approx 2.05$ (нижняя линия) и $Y_{2} / M g \approx 2.69$ (верхняя линия). Эксперименты показывают, что в исследованной области безразмерная подъемная сила не зависит от числа Фруда.

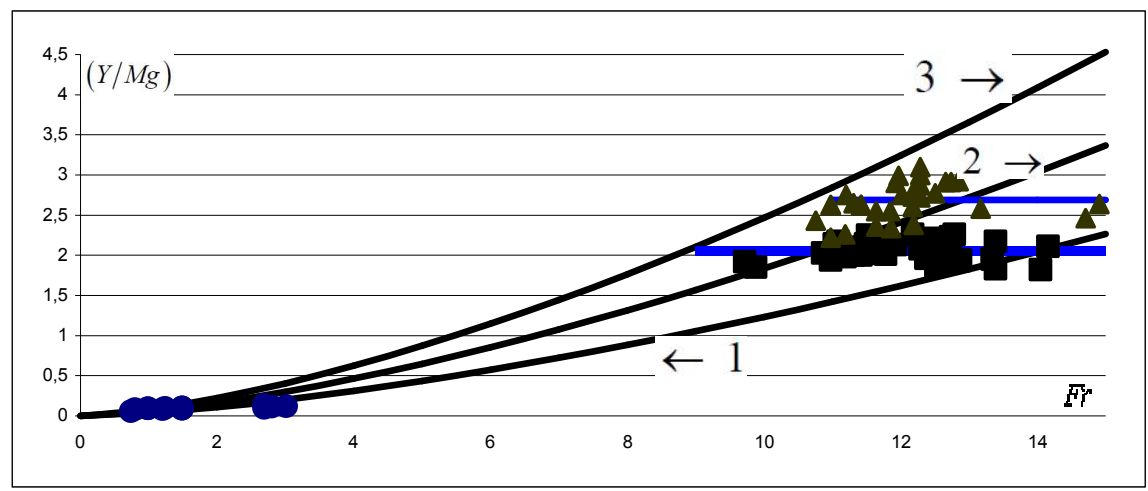

Рис. 4. Зависимость безразмерной подъемной силы $Y / M g=(\Delta / D) F r^{3 / 2} C^{-1}$ от числа Фруда $F r$, и массы системы $(\bullet-m / M \approx 0.12 ; \mathbf{\square}-m / M=2.05$; $\mathbf{\Delta}-m / M=2.69)$

А теперь, пользуясь формулой (3) построим зависимости подъемной силы от числа Фруда для ряда значений безразмерной осадки. На рис. 4 кривая 1 соответствует значению безразмерной осадки $\Delta / D=0.1$, кривая 2 - значению $\Delta / D=0.15$ и кривая $3-\Delta / D=0.2$. При значениях, больших чем $\Delta / D=0.2$, движение становится неустойчивым и незначительное возмущение (например, волна или резкое изменение курсового угла) может перевести качение колес в режим плавания. Кривая 3, по существу, является границей области устойчивого качения. При значениях безразмерной осадки, меньших чем $\Delta / D=0.1$, качение возможно только при достаточной мощности двигателя. В нашем случае скорость модели не превосходила $V \leq 15$ м/с (возможно, потому, что длина разгонного участка была недостаточной). Таким образом, задавая массу колесной системы и пользуясь результатами, приведенными выше (т.е. формулами и графиками), можно определить несущую способность модели (натуры) и минимальную скорость, при которой начинается режим качения. Таблицы опытных данных для безразмерных параметров $\Delta / D, C$, и $Y / M g \approx 2.05$, приведенных на рис. 2, 3, 4 можно найти в работе [8].

А теперь поясним теперь на примерах возможный алгоритм проектирования колесной системы, катящейся по свободной поверхности воды. 
Пример 1. Определить диапазон возможных скоростей качения 4-х колесной полноприводной системы по свободной поверхности воды, если ее масса, диаметр колес и ширина протекторов соответственно равны: $m=3000$ кг, $D=1.5$ м и $B=0.75$ м.

Алгоритм решения.

1) Предполагаем, что форма колес натуры такая же, как и у самоходной модели. Определяем характерную массу системы и еe безразмерную массу по формулам $M=(\pi n / 4) \rho D^{2} B \quad$ и $m / M \quad\left(n=4, \rho=1000\right.$ кг $/ \mathrm{m}^{3}, \quad D=1.5$ м и $\left.B=0.75 \mathrm{м}\right)$. Получаем $M=5298$ кг, $m / M=0.566$.

2) Пользуясь формулой $m / M=\left((\Delta / D) F r^{3 / 2}\right) / C$, и считая, что наибольшая допустимая осадка равна $(\Delta / D)=0.15$, определяем соответствующее число Фруда: $F r=4.55$.

3) Зная число Фруда, находим величину наименьшего допустимого значения скорости: $V=F r \sqrt{g D}=17.45 \mathrm{~m} / \mathrm{c}(62.8$ км/ч), т.е. скорости качения должны быть выше, чем $V=17.45 \mathrm{~m} / \mathrm{c}(62.8$ км/ч). Однако как отмечалось ранее «рабочая» осадка колес составляет примерно $(\Delta / D)=0.1$. Поэтому, проводя аналогичные вычисления при $(\Delta / D)=0.1$, получаем, что в этом случае значение скорости качения равно $V=22.86$ м/с $(82.3$ км/ч) .

Пример 2. Определить диапазон скоростей качения по свободной поверхности воды 8колесной полноприводной системы при той же массе $m=3000$ кг и следующих размерах колес: $D=1$ м и $B=0.5$ м.

Рассуждая так же, как и в предыдущем случае, получаем:

1) Из формулы $M=(\pi n / 4) \rho D^{2} B\left(n=8, \rho=1000 \kappa г / \mathrm{m}^{3}, D=1\right.$ м и $\left.B=0.5 \mathrm{м}\right)$, находим $M=3140$ кг, $m / M=0.955$.

2) Полагая, что $m / M=0.955$ и $(\Delta / D)=0.15$, определяем соответствующее число Фруда: $F r=6.45$.

3) Зная число Фруда, находим минимальное значение скорости качения: $V=F r \sqrt{g D}=20.20 \mathrm{~m} / \mathrm{c}(72.7$ км/ч $)$. Аналогичным образом при осадке колес $(\Delta / D)=0.1$ величина рабочей скорости качения будет равна $V=26.42 \mathrm{M} / \mathrm{c}(95.1 \mathrm{\kappa м} /$ ч).

Два примера, приведенные выше, дают некоторое представление о возможностях колесных систем. Но при достаточной наглядности это всего лишь гипотетические модели. А есть ли среди реальных автомобилей такие, которые без каких-либо конструктивных изменений могут устойчиво катиться по воде? Расчеты показывают, что есть. Это гоночные автомобили Формулы 1. Минимальная масса болида (с пилотом) равна $m=700$ кг, средние значения диаметров и ширины колес соответственно равны: $D \approx 0.665 \mathrm{M}, B \approx 0.350$ м. Следовательно, характерная масса составляет $M \approx 486$ кг., т.е. $m / M=1.44$. Если колеса дополнить небольшими грунтозацепами (как зимнюю резину шипами), то при числе Фруда $F r \approx 11.1$ безразмерная осадка колес при качении по поверхности воды составит примерно $(\Delta / D) \approx 0.1$, т.е. болид будет устойчиво катиться по воде при скорости $V \geq 28.35$ м/с $(102.1 \mathrm{\kappa м} / \mathrm{ч})$.

\section{5. Заключение}

В результате проведенных исследований была разработана методика моделирования явления качения колесного транспортного средства по свободной поверхности воды, предложены схемы измерения осадки цилиндра при буксировке в гидроканале и при качении по воде самоходной модели, получены зависимости безразмерной осадки от числа Фруда при различных значениях безразмерной массы.

Эксперименты показали, что несущая способность колесной системы при большой скорости качения не зависит от числа Фруда. Найдено примерное положение границы области качения, а также установлены условия, при которых транспортное средство может устойчиво катиться по невозмущенной свободной поверхности воды.

Работа выполнена при поддержке гранта РФФИ 16-08-00807. 


\section{Литература}

1. Константинов К.С. Снова по воде на колесах. Катера и яхты №45. Сент.-окт. 1973г. С. 54-56

2. Конов 3.А. По воде на колесах. Катера и яхты №20. авг. 1969г. С.10-14.

3. P.C.Lombardini. R. Fidderman. A New Theory on Propulsion of High Speed Water Craft and its Application to the Hydroller. Proc. 7-th International Congress of Applied Mechanics 2. part II. 1948.

4. R. Taggart. Recent Marine Propulsion Development. "Journal of the American Society of Naval Engineers". May. 1960. V.72. N 2.

5. https://www.youtube.com/watch? $\mathrm{v}=\mathrm{KCMtVawd}$ Vo

6. https://www.youtube.com/watch?v=4oeJjzdlTuI

7. Егоров И.Т. Гидродинамические особенности процесса качения тел по поверхности воды. Докл. XV научно-техн. конф. по теории корабля и гидромех. судна. Апрель 1965. Крыловские чтения. Вып.64. С.170-176.

8. Ерошин В.А., Бойко А.В., Прокофьев В.В. Гидродинамика качения тел по свободной поверхности воды. Изд-во НИИ Механики МГУ. Отчет № 5421, 2018г. 42 с.

9. Седов Л.И. Методы подобия и размерности в механике. Изд. Наука. М.1972. 440 с.

Статья поступила в редакцию 30 августа 2019 г. 